\title{
XXVII. Ueber die krystallographischen Beziehungen einiger Bromderivate des Anhydroecgonins.
}

\author{
Von
}

Arthur Eichengrün in Aachen.

(Mittheilung aus dem mineralogischen Institut der kgl. techn. Hochschule.)

(Mit 3 Textfiguren.)

Vor einiger Zeit berichtete ich an anderer Stelle*) in Gemeinschaft mit. Herrn A. Einhorn uber die bromwasserstoffsauren und salzsauren Salze zweier Bromderivate des Anhydroecgonins, welche wir bei der Darstellung des Dihydrobenzaldehyds erhielten, nämlich des Anhydroecgonindibromids und des $\beta$-Lactons des $\alpha$-Bromecgonins. Diese Salze krystallisiren sämmtlich in zwei verschiedenen Abänderungen, einer wasserhaltigen tetragonalen und einer wasserfreien monoklinen, und zeigen die Glieder je einer dieser Reihen unter sich eine solch' ausserordentliche Aehnlichkeit, dass eine Unterscheidung obne Zuhülfenahme der Schmelzpunktbestimmung nicht möglich ist. Da nun ausserdem ein mit diesen Substanzen chemisch nahe verwandter ebenfalls von uns dargestellter ${ }^{* *}$ ) Körper, das bromwasserstoffsaure Anhydroecgoninhydrobromid, in Ausbildung seiner Krystalle gleichfalls mit den Gliedern der monoklinen Reibe völlig ubereinstimmt, unternahm ich die krystallographische Untersuchung der verschiedenen Salze, um zu entscheiden, ob ihre Krystallformen in ebenso nahem Zusammenhange stehen, wie ihre chemischen Eigenschaften, bezw. welchen morphotropischen Einfluss der Austritt von Bromwasserstoff oder der Umtausch von Brom gegen Wasserstoff in der Seitenkette des Anhydroecgonindibromids auf die Krystallgestalt habe. Die Messungen waren mit grossen Schwierig-

*) Ber. d. d. chem. Ges. 1890, 23, 2870.

**) l. c. $23,2888$. 
keiten verknüpft, da einerseits die monoklinen Krystalle, trotzdem dieseiben in durchweg gut ausgebildeten Exemplaren erhalten wurden, meist infolge ihrer gewölbten oder geknickten Flächen sehr schwankende Werthe ergaben, so dass nur die Mittelwerthe einer sehr grossen Anzahl von Messungen zur Berechnung benutzt werden konnten, andererseits die wasserbaltigen tetragonalen Krystalle so schnell verwitterten und dadurch zur Messung unbrauchbar wurden, dass ich dieselben sofort aus der Lösung auf das Goniometer zu bringen gezwungen war, wobei aber entweder bei ungenugendem Abtrocknen durch anhaftende Mutterlauge falsche Reflexe erhalten, oder bei vollständiger Trocknung die Krystalle durch die strahlende Wärme der Lichtquelle so schnell matt wurden, dass an ein und demselben Exemplare nie mehr als eine Zone durchgemessen werden konnte.

Die erhaltenen Endresultate sind jedoch in Folge der grossen Anzahl gemessener Krystalle hinreichend genau, um uber die oben gestellte Frage befriedigenden Aufschluss zu geben.

\section{Bromwasserstoffsaures Anhydroecgonindibromid, $\mathrm{C}_{5} \mathrm{H}_{7} \mathrm{~N}\left(\mathrm{CH}_{3}\right)-\mathrm{CHBr}-\mathrm{CHBr}-\mathrm{COOH} . \mathrm{HBr}$.}

Es entsteht durch Zersetzung seines, durch Einwirkung von Brom auf Anbydroecgoninchlorhydrat erhaltenen Perbromids, beim Kochen des letzteren mit Alkohol, Eisessig oder besser mit Wasser, unter Durchleiten eines Dampfstromes. Aus der alkoholischen oder essigsauren Lösung werden stets Krystalle des monoklinen Systems, aus der wässerigen Lösung dagegen, je nach Concentration, monokline oder tetragonale Krystalle erhalten, welch' letztere drei Molekule Wasser enthalten.

$$
\begin{gathered}
\text { Tetragonale Form (Hydrat mit } 3 \mathrm{H}_{2} \mathrm{O} \text { ). } \\
\text { Schmelzpunkt } 1820-183^{\circ} . \\
a: c=1: 2,2373 .
\end{gathered}
$$

\begin{tabular}{|c|c|c|}
\hline$p: p=(111):(1 \bar{T} 1)=$ & $\begin{array}{l}\text { Beobachtet: } \\
84^{0} 47^{\prime} 30^{\prime \prime}\end{array}$ & $\begin{array}{l}\text { Grenzwerthe: } \\
84^{0} 44^{\prime}-84^{0} 51^{\prime}\end{array}$ \\
\hline$p: p^{\prime}=(111):(11 \bar{T})=35^{0} \quad 4^{\prime}$ & $35 \quad 10$ & $35 \quad 5-3515$ \\
\hline$p: c=(111):(001)=7228$ & 7221 & $7213-7225$ \\
\hline$\omega: \omega=(557):(5 \overline{5} 7)=8034$ & $80 \quad 22$ & $80 \quad 6-80 \quad 3$ \\
\hline$\omega: \omega^{\prime}=(557):(55 \overline{7})=4746$ & $48 \quad 11$ & $2-4824$ \\
\hline$\omega: c=(557):(001)=66$ & $66 \quad 2$ & $6530-6620$ \\
\hline
\end{tabular}

Die kleineren, 1-2 mm grossen Krystalle sind meist wohlausgebildete Doppelpyramiden, welche häufig durch gleicbzeitiges Auftreten der Flächen von $\{557\}$ gerundet und durch die Endflächen abgestumpft sind. Sie sind 
selten nach beiden Seiten gleichmässig ausgebildet, sondern stellen meist nur an der Spitze abgeschnittene Pyramiden, die auf einer schmalen, durch die antere Pyramide und die Basalfläche gebildeten Platte sitzt, dar. Die grösseren Krystalle, welche bis zu $1 \mathrm{~cm}$ Kantenlänge erhalten wurden, bilden stets stark abgestumpfte Pyramiden oder dünne Platten,

Fig. 1.

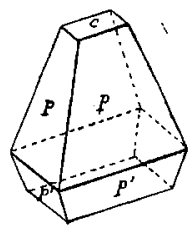

die häufig treppenförmig aufeinander gelagert sind.

Die Spaltbarkeit ist vollkommen nach der Endfläche (001). Das optisch einaxige Interferenzbild ist ungestört; der optische Charakter negativ. Die Krystalle verwittern ausserordentlich leicht an der Luft unter Abgabe von $3 \mathrm{Mol} . \mathrm{H}_{2} \mathrm{O}$. Sie zerfallen hierbei nicht, werden jedoch milchweiss und undurchsichtig. In einem feuchten Präparatenglase aufbewabrt, balten sie sich monatelang unverändert und durchsichtig.

Beobachtete Formen: $c=\{001\}, s=\{101\}, a=\{100\}, r=\{10 \bar{T}\}$, $m=\{110\}, n=\{11 \overline{2}\}$.

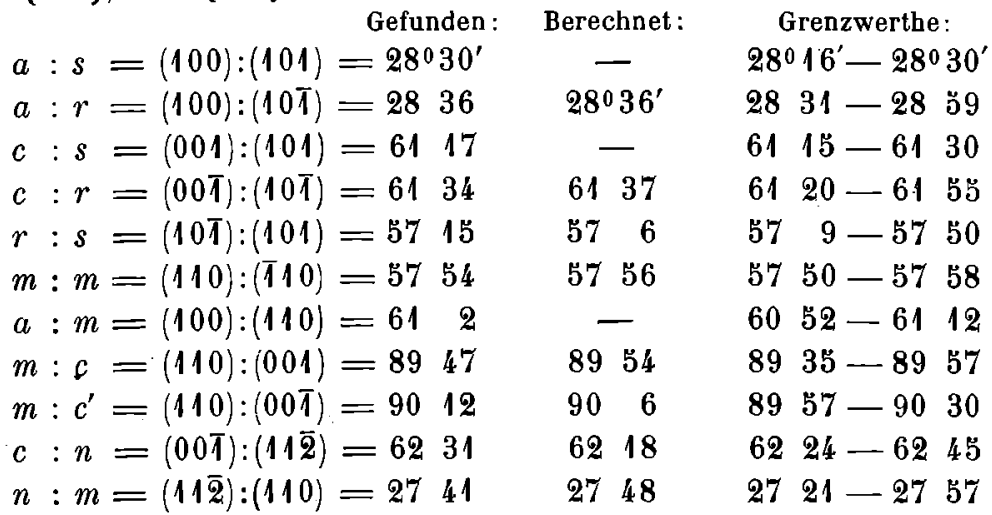

Fig. 2.

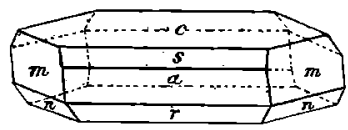

Die farblosen oder durch geringe Mengen Brom schwach gelb gefärbten Krystalle sind gestreckt nach der Axe $b$, häufig flach nach $a\{100\}$, diekleineren meist lang säulenförmig ausgebildet; bei den grösseren ist $n\{1 \mid \overline{2}\}$ häufig mit sämmtlichen Flächen vertreten.

Die Ebene der optischen Axen ist normal zur Symmetrieebene und nahezu parallel der Fläche $c=\{004\}$. Die erste Mittellinie weicht nicht 
merklich von der Axe $a \mathrm{ab}$, so dass in optischer Hinsicht die Krystalle einen durchaus rhombischen Eindruck machen. Bei den grösseren Krystallen sind die ubrigen Querflächen stärker als $a$ ausgebildet und dieselben deshalb zur optischen Untersuchung nicht geeignet. Spaltbarkeit wurde nicht beobachtet.

\section{Salzsanres Anhydroecgonindibromid, $\mathrm{C}_{5} \mathrm{H}_{7} \mathrm{~N}\left(\mathrm{CH}_{3}\right)-\mathrm{CHBr}-\mathrm{CHBr}-\mathrm{COOH} . \mathrm{HCl}$.}

Dasselbe entsteht beim Schutteln der wässerigen Lösung des bromwasserstoffsauren Salzes mit Chlorsilber. Es wurde ebenfalls in zwei Formen erhalten, doch wurde leider der ganze Vorrath an tetragonalen, $3 \mathrm{Mol} . \mathrm{H}_{2} \mathrm{O}$ haltenden Krystallen zu chemiscben $Z$ wecken verbraucht, ehe eine krystallographische Bestimmung möglich war. Ihr Habitus war völlig der gleiche wie der der oben beschriebenen tetragonalen Krystalle; der Schmelzpunkt lag bei $168^{0}-169^{\circ}$.

$$
\begin{gathered}
\text { Monokline Form (wasserfrei). } \\
\text { Schmelzpunkt } 173^{0}-174^{\circ} . \\
a: b: c=1,6003: 1: 3,3924 \\
\beta=89^{\circ} 43^{\prime}
\end{gathered}
$$

Beobachtete Formen: $u=\{102\}, t=\{10 \overline{2}\}, a=\{100\}, m=\{110\}$.

$$
\begin{aligned}
& \text { Berechnet: Beobachtet: Grenzwerthe : } \\
& t: m=(10 \overline{2}):(110)=-67023^{\prime} \quad 67010^{\prime}-67039^{\prime} \\
& t: u=(10 \overline{2}):(102)=-8640 \quad 8631-8649 \\
& m: m=(110):(110)=64^{\circ} \quad 0^{\prime} \quad 64 \quad 8 \quad 6351-6428 \\
& a: m=(100):(110)^{\circ}=-\quad-\quad 58 \quad 0 \quad 5739-5835
\end{aligned}
$$

Der Habitus der Krystalle ist zwar ähnlich dem der vorher beschriebenen monoklinen Form, doch besitzen sie in Folge des vollständigen Fehlens der Fläche $c=\{001\}$ und der unvollkommenen Ausbildung von $a=\{100\}$ eine weit flachere Gestalt. Auch weicht die Axe $a$ stark von den fast ubereinstimmenden Werthen der ubrigen analog zusammengesetzten Bromverbindungen ab. Die Flächen $t$ und $u$ correspondiren nicht mit den Flächen $r=\{10 \pi\}$ und $s=\{101\}$ der letzteren, wie die Winkelwerthe sofort zeigen. Giebt man ihnen jedoch das Symbol $\{10 \overline{2}\}$ bezw. $\{102\}$, so ergiebt sich das obige Axenverhältniss, welches den Axenverhältnissen der ubrigen monoklinen Krystalle entspricht.

Die Krystalle sind farblos, aber tritb und wenig durchsichtig.

Die Ebene der optischen Axen ist die Symmetrieebene. 


\section{Bromwasserstoffsaures $\beta$-Lacton des $\alpha$-Bromecgonins,

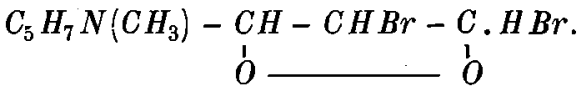

Trägt man in eine kalte, höchst concentrirte Lösung von Kaliumcarbonat in Wasser unter Verreiben ein Salz des Anbydroecgonindibromids in feingepulvertem Zustande ein, saugt die ausfallende weisse Krystallmasse $a b$, löst in wenig Wasser und versetzt mit Bromwasserstoffsäure, so erhält

Fig. 3.

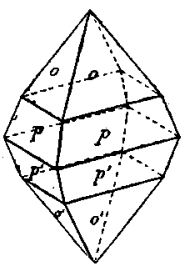
man beim freiwilligen Verdunsten der Lösung tetragonale, 3 Mol. Wasser haltende, beim Einengen der Flussigkeit auf dem Wasserbade dagegen monokline, wasserfreie Krystalle des bromwasserstoffsauren $\beta$-Lactons des $\alpha$-Bromecgonins.

Tetragonale Form (Hydrat mit $3 \mathrm{H}_{2} \mathrm{O}$ ). Schmelzpunkt $210^{0}-2110$. $a: c=1: 2,3620$.

Beobachtete Formen : $c=\{001\}, p=\{111\}, o=\{112\}$.

Berechnet: Beobachtet: Grenzwerthe:

$$
\begin{aligned}
& p: p=(111):(1 \mathrm{~T} 1)=85^{017^{\prime}} \quad 85^{0} 15^{\prime} \quad 85^{0} \quad 4^{\prime}-85^{0} 24^{\prime} \\
& p: p^{\prime}=(111):(11 \overline{1})=3322 \\
& p: c=(111):(001)=7319 \\
& 0: 0=(112):(112)=74 \quad 42 \quad 74 \quad 52 \quad 74 \quad 43-75 \quad 5 \\
& o: o^{\prime}=(112):(11 \overline{2})=6155 \quad 6151 \quad 6143-6155 \\
& o: c=(112):(001)=-\quad \begin{array}{llllll}
59 & 5 & 59 & 0-59 & 7
\end{array}
\end{aligned}
$$

Die Krystalle bilden meist kleine Pyramiden von 1-1 $\frac{1}{2} \mathrm{~mm}$ Kanten-

\begin{tabular}{|c|c|c|}
\hline Berechnet: & Beobachtet: & \\
\hline 1) $=$ & $28^{0} 9^{\prime}$ & $28^{0} 6^{\prime}-28^{\circ} 10^{\prime}$ \\
\hline 01) $=$ & 6130 & $\begin{array}{llll}61 & 21 & -61 & 38\end{array}$ \\
\hline T) $=28^{0} 18^{\prime}$ & $28 \quad 17$ & $28 \quad 10-28 \quad 25$ \\
\hline$=(00 \mathrm{~T}):(10 \mathrm{~T})=62$ & 623 & $6156-62 \quad 11$ \\
\hline
\end{tabular}
länge. Spaltbarkeit war nicht zu bemerken. Die Krystalle sind optisch negativ; das Kreuz des Interferenzbildes öffnet sich kaum.

$$
\begin{gathered}
\text { Monok line Form (wasserfrei). } \\
\text { Schmelzpunkt 2150-2160. } \\
a: b: c=1,8065: 1: 3,3650 \\
\beta=89^{\circ} 39^{\prime} .
\end{gathered}
$$

Beobachtete Formen: $a=\{100\}, c=\{001\}, s=\{101\}, r=\{10 \pi\}$, $m=\{110\}, n=\{11 \overline{2}\}$. 


\begin{tabular}{|c|c|c|}
\hline$m: m=(110):\left(T_{1} 0\right)={ }^{\text {Berechnet: }}$ & $\begin{array}{l}\text { Beobachtet : } \\
57^{0} 56^{\prime}\end{array}$ & $\begin{array}{l}\text { Grenzwerthe: } \\
57^{0} 44^{\prime}-58^{\circ} 13^{\prime}\end{array}$ \\
\hline$a: m=(100):(110)=61^{0} 2^{\prime}$ & 614 & $61 \quad 0-61 \quad 15$ \\
\hline$m: c=(110):(001)=8950$ & 8950 & $8950-895$ \\
\hline$m: c^{\prime}=(110):(00 \bar{T})=9010$ & 9010 & $90 \quad 10-90 \quad 12$ \\
\hline$c^{\prime}: n=(00 \overline{1}):(1 \mid \overline{2})=623930^{\prime \prime}$ & 6232 & $6232-6233$ \\
\hline$n: m=(11 \overline{2}):(110)=273030$ & 2740 & $2738-274$ \\
\hline$n: n=(11 \overline{2}):(\overline{1} 12)=78 \quad 0$ & $78 \quad 29$ & \\
\hline
\end{tabular}

Die Krystalle entsprechen in jeder Hinsicht den monoklinen Formen des bromwasserstoffs. Dibromids, sind jedoch meist durch Ausdehnung in der Richtung der Axe $b$ etwas mehr säulenförmig ausgebildet. Die Ebene der optischen Axen ist, wie dort, fast parallel der Fläche $c=\{001\}$. Die Grösse der durchsichtigen, stark glänzenden Krystalle ist sehr verschieden, es wurden solche von fast mikroskopischer Grösse bis zu $1 \mathrm{~cm}$ Länge und fast $\frac{1}{2} \mathrm{~cm}$ Breite erhalten. Sehr häufig wurden Durchwachsungen zweier oder mehrerer Krystalle beobachtet. Die Fläche $m=\{110\}$ war stets sehr stark ausgebildet, $n=\{1 / \overline{2}\}$ trat nur selten auf.

\section{Salzsaures $\beta$-Lacton des $\alpha$-Bromecgonins, $\mathrm{C}_{5} \mathrm{H}_{7} \mathrm{~N}\left(\mathrm{CH}_{3}\right) \mathrm{CH}-\mathrm{CHBr}-\mathrm{COO} . \mathrm{HCl}$.}

Dasselbe entsteht analog dem bromwasserstoffsauren Salze beim $\mathrm{Zu}-$ satze von Salzsäure zu dem Reactionsproduct aus Anhydroecgonindibromid und Alkalicarbonat. Die tetragonalen Krystalle enthalten ebenfalls $3 \mathrm{Mol}$. $\mathrm{H}_{2} \mathrm{O}$, während die monoklinen wasserfrei sind.

Tetragonale Form (Hydrat mit $3 \mathrm{H}_{2} \mathrm{O}$ ).

Schmelzpunkt 1970-1980.

$$
a: c=1: 2,2850 \text {. }
$$

Beobachtete Formen : $c=\{001\}, o=\{112\}, q=\{778\}$.

\begin{tabular}{|c|c|c|c|c|c|}
\hline$q: q=(778):(7 \overline{7} 8)$ & $\begin{array}{l}\text { Berechnet: } \\
=83^{\circ} 47^{\prime}\end{array}$ & $\begin{array}{l}\text { Beobachtet: } \\
83^{0} 54^{\prime}\end{array}$ & \multicolumn{3}{|c|}{$\begin{array}{c}\text { Grenzwerthe : } \\
83^{0} 39^{\prime}-84^{\circ}\end{array}$} \\
\hline$q: q^{\prime}=(778):(77 \overline{8})$ & $=3858$ & 3927 & 39 & $7-39$ & 47 \\
\hline$q: c=(778):(001)$ & $=70 \quad 31$ & 7020 & 70 & $7-70$ & 35 \\
\hline$o: o=(1 / 2):\left(1 T^{2} 2\right)$ & $=-$ & $735430^{\prime \prime}$ & 73 & $53-73$ & 56 \\
\hline$o: o^{\prime}=(112):(11 \overline{2})$ & $=6332$ & 6316 & 62 & $51-63$ & 40 \\
\hline$o: c=(112):(001)$ & $=5814$ & $58 \quad 24$ & 58 & $10-58$ & 55 \\
\hline$p: p=(111):(1 T 1)$ & $=85$ & - & & - & \\
\hline
\end{tabular}

Die Krystalle sind meist plattenförmig ausgebildet; die kleineren, mehr oktaëderartigen zeigen gewöhnlich die spitze und stumpfe Pyramide und 
die Basis zu gleicher Zeit. Die Spallbarkeit nach $c\{001\}$ ist sehr vollkommen. Der Fläche $o$ wurde das Symbol (112) gegeben, da der entsprechende Werth für die Axe c 1,1425 völlig dem Werthe 1,1810 entspricht, welchen die Fläche $o=\{112\}$ beim bromwasserstoffs. Salze ergiebt. Der Winkel für die nicht auftretende primäre Gestalt $p\{111\}$ berechnet sich alsdann auf $85^{\circ} 2^{\prime}$, der Werth fur $c$ auf 2,2850.

$$
\begin{gathered}
\text { Monokline Form (wasserfrei). } \\
\text { Schmelzpunkt } 202^{0}-203^{0} . \\
a: b: c=1,8250: 1: 3,4780 \\
\beta=88^{0} 57^{\prime} .
\end{gathered}
$$

\begin{tabular}{|c|c|c|c|c|c|c|}
\hline$a: r=$ & $(100):(10 T)$ & $=$ & rechn & & $\begin{array}{c}\text { Beobachtet: } \\
27054^{\prime}\end{array}$ & $\begin{array}{c}\text { Grenzwerthe: } \\
27040^{\prime}-28^{\circ} 12^{\prime}\end{array}$ \\
\hline$: x=$ & $(100):(10.0 .11)$ & $=29$ & & $45^{\prime \prime}$ & $29 \quad 47$ & $2855-30 \quad 17$ \\
\hline$c^{\prime}: r=$ & $(00 \pi):(10 \pi)$ & $=$ & - & & 639 & $6243-6330$ \\
\hline$: x=$ & $(001):(10.0 .11)$ & $=59$ & 13 & 15 & 5910 & $58 \quad 31-6028$ \\
\hline$: x=$ & $(10 \pi):(10.0 .11)$ & $=57$ & 37 & 45 & 5741 & $56 \quad 50-58 \quad 40$ \\
\hline$m: m=$ & $(110):(110)$ & $=57$ & 28 & & 5726 & $57 \quad 2-5745$ \\
\hline$: m=$ & $(100):(110)$ & $=$ & - & & $61 \quad 16$ & $6049-61 \quad 23$ \\
\hline$: c=$ & $(110):(001)$ & $=89$ & 29 & 40 & 8930 & $89 \quad 20-89 \quad 45$ \\
\hline$: y=$ & $(001):$ & $=44$ & 7 & & 4346 & $4311-4356$ \\
\hline$y: r=$ & $(10 \overline{2}):$ & $=19$ & 2 & & 1836 & $1810-1922$ \\
\hline$x: z=1$ & .0 .11 & $=13$ & & 45 & 144 & $1427-15 \quad 3$ \\
\hline$: z=$ & 100 & $=46$ & & 30 & 4625 & $46 \quad 10-46 \quad 35$ \\
\hline$: n=$ & & $=76$ & 26 & & 7724 & $7712-7730$ \\
\hline$: y=$ & $(11 \overline{2}):(10 \overline{2})$ & $=51$ & 47 & & 5120 & $0-5126$ \\
\hline
\end{tabular}

Beobachtete Formen: $a=\{100\}, c=\{001\}, x=\{10.0 .11\}, r=$ $\{10 T\}, y=\{10 \overline{2}\}, z=\{509\}, m=\{110\}, n=\{11 \overline{2}\}$.

Die grösseren Krystalle zeigen nur die Flächen $a, c, r, x$ und $m$, die kleineren dagegen auch die ubrigen oben angegebenen Flächen, doch ist beim Auftreten der Querflächen $y$ und $z$ die Fläche $a$ niemals, beim Auftreten von $n$ das Prisma $m$ nur in einem Falle beobachtet worden. Da die Krystalle eine ausgeprägt säulenförmige Ausbildung zeigten, waren die kleineren nadelförmigen Exemplare, an welchen die Nebenflächen beobachtet wurden, durch das gleichzeitige Auftreten der vielen Querflächen stark gerundet und ergaben deshalb, wie die weit auseinander liegenden Grenzwerthe zeigen, schlechte Messungsresultate für die Nebenflächen.

Es ist demnach nicht ausgeschlossen, dass den Flächen $z$ und $y$ ein

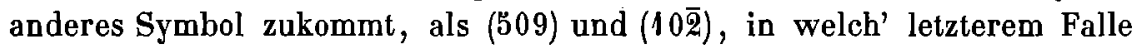
auch $n$ nicht $=(11 \overline{2})$ sein würde. Die Krystalle sind meist buschelförmig verwachsen, ihre Grösse schwankt von Haaresdicke bis zu $1 \mathrm{~mm}$ Durchmesser in der Richtung der Axe $c$. Sie haben ausserordentlich starkes Licht- 
brechungsvermögen; ihr optisches Verhalten stimmt mit dem des bromwasserstoffsauren Salzes uberein, d. h. optische Axenebene fast genau der Ebene $c\{001\}$ entsprechend.

\section{Bromwasserstoffsaures Anhydroecgoninhydrobromid, $\mathrm{C}_{9} \mathrm{H}_{14} \mathrm{NO}_{2} \mathrm{Br} . \mathrm{HBr}$.}

Dieser Körper entsteht bei sechs- bis siebentägiger Einwirkung von Bromwasserstoffeisessig auf Anbydroecgonin unter Druck und bei einer Temperatur von $100 \%$. Es ist noch nicht entschieden, ob ihm die Formel

oder

$$
\mathrm{C}_{5} \mathrm{H}_{7} \mathrm{~N}\left(\mathrm{CH}_{3}\right)-\mathrm{CH}_{2}-\mathrm{CHBr}-\mathrm{COOH} . \mathrm{HBr}
$$

zuzuschreiben ist. Wasserhaltig wurde derselbe nicht erhalten. Die wasserfreien monoklinen Krystalle zeigen den Habitus der oben bescbriebenen Körper, machen jedoch einen mehr rhombischen Eindruck und zeigen fast stets Skelettbildung im Innern. Schmelzpunkt $250^{\circ}$.

$$
\begin{gathered}
a: b: c=1,8229: 1: 3,3156 ; \\
\beta=89^{\circ} 44^{\prime} 30^{\prime \prime} .
\end{gathered}
$$

\begin{tabular}{|c|c|c|}
\hline$a: q=(100):(103)=58^{0} 38^{\prime}$ Berechnet: & $\begin{array}{l}\text { Beobachtet : } \\
58^{0} 34^{\prime}\end{array}$ & $\begin{array}{c}\text { Grenzwerthe: } \\
58^{0} 26^{\prime}-58^{\circ} 44^{\prime}\end{array}$ \\
\hline$a: v=(100):(10 \overline{3})=5857$ & 591 & $5850-5916$ \\
\hline$q: v=(103):(10 \overline{3})=-$ : & 6225 & $6213-6235$ \\
\hline$a: m=(100):(110)=-$ & 6115 & $6023-6155$ \\
\hline$m: m=(110):(1 \overline{1} 0)=5830$ & $58 \quad 40$ & $5810-5913$ \\
\hline$q: m=(103):(110)=-$ & 7530 & $7527-7532$ \\
\hline$a: s=(100):(101)=2852$ & - & - \\
\hline
\end{tabular}

Beobachtete Formen: $a=\{100\}, q=\{103\}, v=\{10 \overline{3}\}, m=\{110\}$.

Die Domenflächen $q$ und $v$ entsprechen, wie die Winkel zeigen, nicht den Flächen $s=\{101\}$ und $r=\{10 \bar{T}\}$ des Dibromids, ihr Symbol ist vielmehr $\{103\}$ bezw. $\{10 \overline{3}\}$.

Die Fläche $c=\{001\}$, sowie Pyramidenflächen wurden nicht beobachtet. Die Ebene der optischen Axen ist die Symmetrieebene. Die erste Mittellinie ist fast normal zur Querfläche $\{100\}$.

Die morphotropischen Beziehungen zwischen den verschiedenen Körpern werden durch folgende Vergleichstabelle dargelegt.

Hydrate mit $3 \mathrm{H}_{2} \mathrm{O}$.

$\begin{array}{ccccc}C_{5} H_{7} N\left(\mathrm{CH}_{3}\right)- & \text { Axenverhältuiss : } & (111):(1 \overline{1} 1) & \text { Schmelzp.: } & \text { Differenz: } \\ -\mathrm{CHBr}-\mathrm{CHBr}-\mathrm{COOH} . \mathrm{HCl} & - & - & 1690 & \\ -\mathrm{CHBr}-\mathrm{CHBr}-\mathrm{COOH} . \mathrm{HBr} & 1: 2,2373 & 84048^{\prime} & 183 & 140 \\ -\mathrm{CH}-\mathrm{CHBr}-\mathrm{COO} . \mathrm{HCl} & 1: 2,2850 & 85 \quad 2 & 197 & 14 \\ -\mathrm{CH}-\mathrm{CHBr-COO} . \mathrm{HBr} & 1: 2,3620 & 8517 & 211 & 14\end{array}$


382 Arthur Eichengrün. Ueber die krystallogr. Beziehungen einiger Bromderivate.

Wasserfreie Körper.

\begin{tabular}{|c|c|c|c|c|c|c|c|}
\hline $\mathrm{C}_{5} \mathrm{H}_{7} \mathrm{~N}\left(\mathrm{CH}_{3}\right)-$ & Axenverbältniss: & $\beta:$ & $\begin{array}{l}(110): \\
(T 10)\end{array}$ & $\begin{array}{l}(100): \\
(110)\end{array}$ & $\begin{array}{l}(100): \\
(101)\end{array}$ & Schmp: : & : Diff.: \\
\hline$-\mathrm{CHBr}-\mathrm{CHBr}-\mathrm{COOH}, \mathrm{HCl}$ & $4,6003: 1: 3,3924$ & $89043^{\prime}$ & & & & 1740 & \\
\hline$-\mathrm{CHBr}-\mathrm{CHBr}-\mathrm{COOH} . \mathrm{HBr}$ & 1,8065:1:3,9204 & 8947 & $57056^{\prime}$ & $6102^{\prime}$ & $28036^{\prime}$ & 188 & 140 \\
\hline$-\mathrm{CH}-\mathrm{CHBr}-\mathrm{COO} . \mathrm{HCl}$ & $1,8250: 1: 3,4780$ & 8857 & 5728 & 6116 & 2754 & 202 & 14 \\
\hline$-\mathrm{CH}-\mathrm{CHBr}-\mathrm{COO} \cdot \mathrm{HBr}$ & $1,8065: 1: 3,3650$ & 8939 & 5756 & $61 \quad 2$ & 28 & 216 & 14 \\
\hline$-\mathrm{CHBr}-\mathrm{CH} \mathrm{H}_{2} \mathrm{COOH} \cdot \mathrm{HBr}$ & $1,8229: 1: 3,3156$ & 8941,5 & 5830 & 6115 & 2852 & 250 & 34 \\
\hline
\end{tabular}

Es zeigt sich auf den ersten Blick, in welch' engem Zusammenhange die Krystallformen der verschiedenen Salze stehen, deren chemische Verwandtschaft sich auch durch das auffallend proportionale Aufsteigen der Schmelzpunkte kundgiebt. Eine ähnliche Proportionalität zeigt sich auch bei der Krystallform der tetragonalen Reihe, da die Werthe für die Axe $a$ gleichmässig um circa 0,06, fur den Winkel (111):(1T1) um circa 15' aufsteigen. Bei den monoklinen Formen der wasserfreien Körper ist zwar eine eigentliche Proportionalitäl nicht zu bemerken, doch ist die Variation del Krystallform, welche die chemische Aenderung im Molekül hervorbringt, mit Ausnahme des Werthes für $a$ beim salzsauren Dibromid eine sehr geringe, so dass, bei gleicher Ausnahme, die Schwankungen der Axe a 0,018, der Axe $c 0,1624$ und des $\Varangle \beta 0^{0} 49^{\prime}$ nicht übersteigen. Noch geringer sind die Schwankungen der Winkel, welche die von den Aenderungen des Winkels $\beta$ nicht beeinflusste Fläche $m$ mit den Pinakoid- und Pyramidenflächen bildet, also die Winkel $m: m, a: m, c: m$ und $n: m$. Bei der grossen, theils durch Interferenzerscheinungen, theils durch unvollkommene Ausbildung und Abrundung der Krystalle hervorgerufenen Ungenauigkeit mancher erhaltener Werthe, deren Grenzwerthe oft um $10-2^{0}$ auseinander liegen, ist es nicht ausgeschlossen, dass die Aehnlichkeit der Krystall form noch eine viel grössere ist. Jedenfalls genügen aber die angeführten Zahlen, um zu zeigen, dass der Austausch oder Austritt von Brom oder Bromwasserstoff in der Seitenkette des Anhydroecgonindibromids trotz der verhältnissmässig grossen Aenderung der Molekulargrösse von sehr geringem Einflusse auf die Krystallform ist. 